\title{
POINTWISE CONVERGENCE OF MARCINKIEWICZ-FEJÉR MEANS OF DOUBLE VILENKIN-FOURIER SERIES
}

\author{
USHANGI GOGINAVA
}

\begin{abstract}
In this paper we give a characterization of points in which Marcinkiewicz-Fejér means of double Vilenkin-Fourier series converges.
\end{abstract}

\section{INTRODUCTION}

Lebesgue's [14 theorem is well known for trigonometric Fourier series: the Fejér means $\sigma_{n} f$ of $f$ converge to $f$ almost everywhere if $f \in L_{1}([0,2 \pi))$ (see also Zygmund [26]). The analogous result for Walsh-Fourier series is due to Fine [3]. Later Schipp [19] showed that the maximal operator $\sigma^{*}$ of the Fejér means of the one-dimensional Walsh-Fourier series is of weak type $(1,1)$, from which the a. e. convergence follows by standard arguments.

Marcinkievicz [15] verified for two-dimensional trigonometric Fourier series that the Marcinkiewicz-Fejér means

$$
\sigma_{n}(f)=\frac{1}{n} \sum_{j=1}^{n} S_{j, j}(f)
$$

of a function $f \in L \log L([0,2 \pi) \times[0,2 \pi))$ converge a.e. to $f$ as $n \rightarrow \infty$, where $S_{j, j}(f)$ denotes the cubic partial sums of the Fourier series of $f$. Later Zhizhiashvili [24, 25] extended this result to all $f \in L_{1}([0,2 \pi) \times[0,2 \pi))$. The analogous result for two-dimensional Walsh-Fourier series is due to Weisz 21].

In the one-dimensional case the set of convergence is characterized with the help of Lebesgue points. It is known that a.e. point $x \in[0,2 \pi)$ is a Lebesgue point of $f \in L_{1}([0,2 \pi))$ and the Fejér means of the trigonometric Fourier series of $f \in L_{1}([0,2 \pi))$ converge to $f$ at each Lebesgue point (see Butzer and Nessel [2]). Weisz [20] introduced the notion of Walsh-Lebesgue points and proved the analogous results for Walsh-Fourier series.

For Vilenkin-Fourier series the author and Gogoladze [11] introduced the notion of Vilenkin-Lebesgue points and proved that Fejér means of the

${ }^{0} 2010$ Mathematics Subject Classification. 42C10.

Key words and phrases: Vilenkin function, Pointwise summability, Marcinkiewicz-Fejér means, Lebesgue points.

The research was supported by Shota Rustaveli National Science Foundation grant no.DI/9/5-100/13 (Function spaces, weighted inequalities for integral operators and problems of summability of Fourier series) 
Vilenkin-Fourier series of $f \in L_{1}\left(G_{m}\right)$ converges to $f$ at each VilenkinLebesgue points. In this paper we will generalize these results for the Marcinkiewicz-Fejér means of double Vilenkin-Fourier series and characterize the set of convergence of these means. We introduce the MarcinkiewiczLebesgue points and prove that a.e. point is a Marcinkiewicz-Lebesgue point of an integrable function $f$ and the Marcinkiewicz-Fejér means of the double Vilenkin-Fourier series of $f$ converge to $f$ at each Marcinkiewicz-Lebesgue point.

The problems of summability of cubical partial sums of multiple Fourier series have been investigated in ([4]-[13]).

\section{Definitions and Notation}

Let $\mathbb{N}_{+}$denote the set of positive integers, $\mathbb{N}:=\mathbb{N}_{+} \cup\{0\}$. Let $m:=$ $\left(m_{0}, m_{1}, \ldots\right)$ denote a sequence of positive integers not less than 2. Denote by $Z_{m_{k}}:=\left\{0,1, \ldots, m_{k}-1\right\}$ the additive group of integers modulo $m_{k}$. Define the group $G_{m}$ as the complete direct product of the groups $Z_{m_{j}}$, with the product of the discrete topologies of $Z_{m_{j}}$ 's. The direct product $\mu$ of the measures

$$
\mu_{k}(\{j\}):=\frac{1}{m_{k}} \quad\left(j \in Z_{m_{k}}\right)
$$

is the Haar measure on $G_{m}$ with $\mu\left(G_{m}\right)=1$. If the sequence $m$ is bounded, then $G_{m}$ is called a bounded Vilenkin group. The elements of $G_{m}$ can be represented by sequences $x:=\left(x_{0}, x_{1}, \ldots, x_{j}, \ldots\right),\left(x_{j} \in Z_{m_{j}}\right)$. The group operation + in $G_{m}$ is given by $x+y=\left(x_{0}+y_{0}\left(\bmod m_{0}\right), \ldots, x_{k}+y_{k}\left(\bmod m_{k}\right), \ldots\right)$ , where $x=\left(x_{0}, \ldots, x_{k}, \ldots\right)$ and $y=\left(y_{0}, \ldots, y_{k}, \ldots\right) \in G_{m}$. The inverse of + will be denoted by -. In this paper we will consider only bounded Vilenkin group.

It is easy to give a base for the neighborhoods of $G_{m}$ :

$$
\begin{gathered}
I_{0}(x):=G_{m}, \\
I_{n}(x):=\left\{y \in G_{m} \mid y_{0}=x_{0}, \ldots, y_{n-1}=x_{n-1}\right\}
\end{gathered}
$$

for $x \in G_{m}, n \in \mathbb{N}$. Define $I_{n}:=I_{n}(0)$ for $n \in \mathbb{N}_{+}$. Set $e_{n}:=(0, \ldots, 0,1,0, \ldots) \in$ $G_{m}$ the $n$th coordinate of which is 1 and the rest are zeros $(n \in \mathbb{N})$.

If we define the so-called generalized number system based on $m$ in the following way: $M_{0}:=1, M_{k+1}:=m_{k} M_{k}(k \in \mathbb{N})$, then every $n \in \mathbb{N}$ can be uniquely expressed as $n=\sum_{j=0}^{\infty} n_{j} M_{j}$, where $n_{j} \in Z_{m_{j}}\left(j \in \mathbb{N}_{+}\right)$and only a finite number of $n_{j}$ 's differ from zero. We use the following notation. Let (for $n>0$ ) $|n|:=\max \left\{k \in \mathbb{N}: n_{k} \neq 0\right\}$ (that is, $M_{|n|} \leq n<M_{|n|+1}$ ).

Next, we introduce on $G_{m}$ an orthonormal system which is called the Vilenkin system. At first define the complex valued functions $r_{k}(x): G_{m} \rightarrow$ $\mathbb{C}$, the generalized Rademacher functions in this way

$$
r_{k}(x):=\exp \left(\frac{2 \pi \imath x_{k}}{m_{k}}\right)\left(\imath^{2}=-1, x \in G_{m}, k \in \mathbb{N}\right) .
$$


It is known that

$$
\sum_{i=0}^{m_{n}-1} r_{n}^{i}(x)=\left\{\begin{array}{l}
0, \text { if } x_{n} \neq 0 \\
m_{n}, \text { if } x_{n}=0
\end{array} .\right.
$$

Now define the Vilenkin system $\psi:=\left(\psi_{n}: n \in \mathbb{N}\right)$ on $G_{m}$ as follows:

$$
\psi_{n}(x):=\prod_{k=0}^{\infty} r_{k}^{n_{k}}(x) \quad(n \in \mathbb{N}) \text {. }
$$

Specifically, we call this system the Walsh-Paley one if $m \equiv 2$.

The Vilenkin system is orthonormal and complete in $L^{1}\left(G_{m}\right)[1]$.

We consider the double system $\left\{\psi_{n}(x) \times \psi_{m}(y): n, m \in \mathbb{N}\right\}$ on $G_{m} \times G_{m}$. The notation $a \lesssim b$ in the whole paper stands for $a \leq c b$, where $c$ is an absolute constant.

The rectangular partial sums of the double Vilenkin-Fourier series are defined as follows:

$$
S_{M, N}(x, y ; f):=\sum_{i=0}^{M-1} \sum_{j=0}^{N-1} \widehat{f}(i, j) \psi_{i}(x) \psi_{j}(y),
$$

where the number

$$
\widehat{f}(i, j)=\int_{G_{m} \times G_{m}} f(x, y) \psi_{i}(x) \psi_{j}(y) d \mu(x, y)
$$

is said to be the $(i, j)$ th Vilenkin-Fourier coefficient of the function $f$.

The norm (or quasinorm) of the space $L_{p}\left(G_{m} \times G_{m}\right)$ is defined by

$$
\|f\|_{p}:=\left(\int_{G_{m} \times G_{m}}|f(x, y)|^{p} d \mu(x, y)\right)^{1 / p} \quad(0<p<+\infty) .
$$

The space weak- $L_{p}\left(G_{m} \times G_{m}\right)$ consists of all measurable functions $f$ for which

$$
\|f\|_{\text {weak }-L_{p}\left(G_{m} \times G_{m}\right)}:=\sup _{\lambda>0} \lambda \mu(|f|>\lambda)^{1 / p}<+\infty .
$$

The $\sigma$-algebra generated by the dyadic 2 -dimensional $I_{k} \times I_{k}$ cube of measure $M_{k}^{-1} \times M_{k}^{-1}$ will be denoted by $F_{k}(k \in \mathbb{N})$. Denote by $f=\left(f^{(n)}, n \in \mathbb{N}\right)$ one-parameter martingales with respect to $\left(F_{n}, n \in \mathbb{N}\right.$ ) (for details see, e. g. [22]). The maximal function of a martingale $f$ is defined by

$$
f^{*}=\sup _{n \in \mathbb{N}}\left|f^{(n)}\right| .
$$

In case $f \in L_{1}\left(G_{m} \times G_{m}\right)$, the maximal function can also be given by

$$
f^{*}(x, y)=\sup _{n \in \mathbb{N}} \frac{1}{\mu\left(I_{n}(x) \times I_{n}(y)\right)}\left|\int_{I_{n}(x) \times I_{n}(y)} f(t, u) d \mu(t, u)\right|,
$$




$$
(x, y) \in G_{m} \times G_{m} .
$$

For $0<p<\infty$ the dyadic martingale Hardy space $H_{p}(G \times G)$ consists of all martingales for which

$$
\|f\|_{H_{p}}:=\left\|f^{*}\right\|_{p}<\infty .
$$

If $f \in L_{1}\left(G_{m} \times G_{m}\right)$ then it is easy to show that the sequence $\left(S_{M_{n}, M_{n}}(f): n \in \mathbb{N}\right)$ is a martingale. If $f$ is a martingale, that is $f=\left(f^{(0)}, f^{(1)}, \ldots\right)$ then the Vilenkin-Fourier coefficients must be defined in a little bit different way:

$$
\widehat{f}(i, j)=\lim _{k \rightarrow \infty} \int_{G_{m} \times G_{m}} f^{(k)}(x, y) \psi_{i}(x) \psi_{j}(y) d \mu(x, y) .
$$

The Vilenkin-Fourier coefficients of $f \in L_{1}\left(G_{m} \times G_{m}\right)$ are the same as the ones of the martingale $\left(S_{M_{n}, M_{n}}(f): n \in \mathbb{N}\right)$ obtained from $f$.

For $n=1,2, \ldots$ and a martingale $f$ the Marcinkiewicz-Fejér means of order $n$ of the 2-dimensional Vilenkin-Fourier series of the function $f$ is given by

$$
\sigma_{n}(x, y ; f)=\frac{1}{n} \sum_{j=0}^{n-1} S_{j, j}(x, y ; f)
$$

If

$$
K_{n}(x, y):=\frac{1}{n} \sum_{k=0}^{n-1} D_{k}(x) D_{k}(y)
$$

denotes the 2-dimensional Marcinkiewicz-Fejér kernel of order $n$ then

$$
\sigma_{n}(x, y ; f)=\int_{G_{m} \times G_{m}} f(t, u) K_{n}(x-t, y-u) d \mu(t, u) .
$$

A bounded measurable function $a$ is a p-atom, if there exists a generalized square $I \times J \in F_{n}$, such that

a) $\int_{I \times J} a d \mu=0$;

b) $\|a\|_{\infty} \leq \mu(I \times J)^{-1 / p}$;

c) $\operatorname{supp} a \subset I \times J$.

An operator $T$ which maps the set of martingales into the collection of measurable functions will be called p-quasi-local if there exist a constant $C_{p}>0$ such that for every p-atom $a$

$$
\int_{G_{m} \times G_{m} \backslash(I \times J)}|T a|^{p} \leq C_{p}<\infty,
$$

where $I \times J$ is the support of the atom. 


\section{MarCinkieWiCZ-LeBesgue POINTS}

In the one-dimensional case a point $x \in(-\infty, \infty)$ is called a Lebesgue point of a function $f$ if

$$
\lim _{h \rightarrow 0} \frac{1}{h} \int_{0}^{h}|f(x+t)-f(x)| d t=0 .
$$

It is known that a.e. point $x \in[0,2 \pi)$ is a Lebesgue point of $f \in L_{1}([0,2 \pi))$ and that the Fejér means of the trigonometric Fourier series of $f \in L_{1}([0,2 \pi))$ converge to $f$ at each Lebesgue point (see Butzer and Nessel [2]). Feichtinger and Weisz [18] extended these results to two-dimensional trigonometric Fourier series, to arbitrary summability methods and to all $f \in$ $L(\log L)^{+}\left([0,2 \pi)^{2}\right)$.

Weisz introduced the one-dimensional Walsh-Lebesgue point in [20]: $x \in$ $G_{2}$ is a Walsh-Lebesgue point of $f \in L_{1}\left(G_{2}\right)$, if

$$
\lim _{n \rightarrow \infty} \sum_{k=0}^{n} 2^{k} \int_{I_{n}\left(e_{k}\right)}|f(x+t)-f(x)| d t=0 .
$$

He proved that a.e. point $x \in G_{2}$ is a Walsh-Lebesgue point of an integrable function $f$. Moreover, the Fejér means of the Walsh-Fourier series of $f \in$ $L_{1}\left(G_{2}\right)$ converge to $f$ at each Walsh-Lebesgue point. The higher dimensional extension of this result can be found in [23, 11].

In [11] it is characterized the set of convergence of Vilenkin-Fejér means. We introduced the operator

$$
W_{A} f(x):=\sum_{s=0}^{A-1} M_{s} \sum_{r_{s}=1}^{m_{s}-1} \int_{I_{A}\left(x-r_{s} e_{s}\right)}|f(t)-f(x)| d \mu(t) .
$$

A point $x \in G_{m}$ is a Vilenkin-Lebesgue point of $f \in L_{1}\left(G_{m}\right)$, if

$$
\lim _{A \rightarrow \infty} W_{A} f(x)=0 .
$$

The following are proved in [11].

Theorem GG ([11]). Let $f \in L_{1}\left(G_{m}\right)$, where $G_{m}$ is a bounded Vilenkin group. Then

$$
\lim _{n \rightarrow \infty} \sigma_{n} f(x)=f(x)
$$

for all Vilenkin-Lebesgue points of $f$.

Corollary GG1 ([1] ). Let $f \in L_{1}\left(G_{m}\right)$, where $G_{m}$ is a bounded Vilenkin group. Then

$$
\lim _{A \rightarrow \infty} W_{A} f(x)=0 \text { for a. e. } x \in G_{m},
$$

thus a. e. point is a Vilenkin-Lebesgue point of $f$. 
Corollary GG2 ([11]). Let $f \in L_{1}\left(G_{m}\right)$, where $G_{m}$ is a bounded Vilenkin group. Then

$$
\sigma_{n}(x ; f) \rightarrow f(x) \text { for a.e. } x \in G_{m} .
$$

For two-dimensional Walsh-Fourier series Weisz [21] has proved that for all $f \in L_{1}(G \times G)$ the Marcinkiewicz-Fejér means $\sigma_{n} f$ converge a.e. to $f$ as $n \rightarrow \infty$.

In [13] it is characterized the set of convergence of Marcinkiewicz-Fejér means of two-dimensional Walsh-Fourier series.

For two-dimensional Vilenkin-Fourier series Gat [4] has proved that for all $f \in L_{1}\left(G_{m} \times G_{m}\right)$ the Marcinkiewicz-Fejér means $\sigma_{n} f$ converge a.e. to $f$ as $n \rightarrow \infty$.

In this paper we will characterize the set of convergence of MarcinkiewiczFejér means with respect to bounded Vilenkin system. We introduce the Marcinkiewicz-Lebesgue points and prove that a.e. point is a MarcinkiewiczLebesgue point of an integrable function $f$ and the Marcinkiewicz-Fejér means of the two-dimensional Vilenkin-Fourier series of $f$ converge to $f$ at each Marcinkiewicz-Lebesgue point.

Set

$$
\begin{gathered}
W_{j}(x, y ; f):=M_{j}^{-1} \sum_{q=0}^{j-1} \sum_{k=q}^{j-1} \sum_{I_{q}=1}^{m_{q}-1} M_{q} M_{k}^{2} \\
\times \int_{I_{k} \times I_{k}\left(u_{q} e_{q}\right)}|f(x-t, y-u)-f(x, y)| r_{k+1, j-1}(t, u) d \mu(t, u) \\
+M_{j}^{-1} \sum_{q=0}^{j-1} \sum_{k=q}^{j-1} \sum_{t_{q}=1}^{m_{q}-1} M_{q} M_{k}^{2} \\
\times \int_{I_{k}\left(t_{q} e_{q}\right) \times I_{k}}+\sum_{s=0}^{j} \sum_{i=s}^{j} M_{s} M_{i} \sum_{u_{s}=1}^{m_{s}-1} \\
\times \int_{I_{j} \times I_{i}\left(u_{s} e_{s}\right)}|f(x-t, y-u)-f(x, y)| r_{k+1, j-1}(t, u) d \mu(t, u) \\
\times \int_{I_{i}\left(t_{s} e_{s}\right) \times I_{j}}|f(x, y)| d \mu(t, u) \\
|f(x-t, y-u)-f(x, y)| d \mu(t, u),
\end{gathered}
$$


where

$$
r_{i, n}(x, y):=\prod_{l=i}^{n}\left(\sum_{s=0}^{m_{l}-1} \psi_{M_{l}}^{s}(x+y)\right) .
$$

By (11) it is easy to show that

$$
r_{i, n}(x, y)=\left\{\begin{array}{l}
m_{i} m_{i+1} \cdots m_{n}, x_{j}+y_{j}\left(\bmod m_{j}\right)=0, j=i, i+1, \ldots, n \\
0, \text { otherwise }
\end{array} .\right.
$$

A point $(x, y) \in G_{m} \times G_{m}$ is a Marcinkiewicz-Lebesgue point (for bounded Vilenkin group) of $f \in L_{1}\left(G_{m} \times G_{m}\right)$, if

$$
\lim _{n \rightarrow \infty} W_{n}(x, y ; f)=0 \text {. }
$$

Set

$$
\begin{gathered}
V_{n}(x, y ; f):=\sum_{q=0}^{n-1} \sum_{k=q}^{n-1} \sum_{t_{q}=1}^{m_{q}-1} \frac{M_{q} M_{k}}{m_{k}} \\
\times \int_{I_{k}\left(t_{q} e_{q}\right) \times I_{k}} f(x-t, y-u) \mathbb{I}_{\left\{t_{r}+u_{r}\left(\bmod m_{r}\right)=0, r=k+1, \ldots, n-1\right\}}(t, u) d \mu(t, u)
\end{gathered}
$$$$
+\sum_{q=0}^{n-1} \sum_{k=q}^{n-1} \sum_{u_{q}=1}^{m_{q}-1} \frac{M_{q} M_{k}}{m_{k}}
$$$$
\times \int_{I_{k} \times I_{k}\left(u_{q} e_{q}\right)} f(x-t, y-u) \mathbb{I}_{\left\{t_{r}+u_{r}\left(\bmod m_{r}\right)=0, r=k+1, \ldots, n-1\right\}}(t, u) d \mu(t, u) d \mu(t, u)
$$

$$
\begin{gathered}
+\sum_{s=0}^{n} \sum_{i=s}^{n} M_{s} M_{i} \sum_{u_{s}=1}^{m_{s}-1} \\
\times \int_{I_{n} \times I_{i}\left(u_{s} e_{s}\right)} f(x-t, y-u) d \mu(t, u) \\
+\sum_{s=0}^{n} \sum_{i=s}^{n} M_{s} M_{i} \sum_{t_{s}=1}^{m_{s}-1} \\
\int_{I_{i}\left(t_{s} e_{s}\right) \times I_{n}} f(x-t, y-u) d \mu(t, u) \\
:=\sum_{j=1}^{4} V_{n}^{(j)}(x, y ; f),
\end{gathered}
$$

where $\mathbb{I}_{E}$ is characteristic function of the set $E$.

It is easy to see that $W_{n} f(x, y) \rightarrow 0$ as $n \rightarrow \infty$ if and only if

$$
\lim _{n \rightarrow \infty} V_{n}(|f-f(x, y)|)(x, y)=0 .
$$


Let

$$
V f:=\sup _{n}\left|V_{n} f\right|, \quad V^{(i)} f:=\sup _{n}\left|V_{n}^{(i)} f\right|, i=1,2,3.4 .
$$

\section{Main Results}

In this paper we prove that the following are true

Theorem 1. Let $f \in L_{1}\left(G_{m} \times G_{m}\right)$. Then

$$
\lim _{n \rightarrow \infty} \sigma_{n}(x, y ; f)=f(x, y)
$$

for all Marcinkiewicz-Lebesgue points of $f$.

Theorem 2. Let $p>1 / 2$. Then

$$
\|V f\|_{p} \leq c_{p}\|f\|_{p} \quad\left(f \in H_{p}\left(G_{m} \times G_{m}\right)\right)
$$

and

$$
\sup _{\lambda} \lambda \mu\{V f>\lambda\} \leq c\|f\|_{1} .
$$

It is easy to show that $\lim _{n \rightarrow \infty} W_{n}(x, y ; f)=0$ for every Vilenkin polynomials and $(x, y) \in G_{m} \times G_{m}$. Since the Vilenkin polynomials are dense in $L_{1}\left(G_{m} \times\right.$ $G_{m}$ ), Theorem 2 and the usual density argument (see Marcinkiewicz and Zygmund [16]) imply

Corollary 1. Let $f \in L_{1}\left(G_{m} \times G_{m}\right)$. Then

$$
\lim _{n \rightarrow \infty} W_{n}(x, y ; f)=0 \quad \text { a. e. }(x, y) \in G_{m} \times G_{m},
$$

thus a. e. points is a Marcinkiewicz-Lebesgue point of $f$.

Corollary 2. (Gat [4]) Let $f \in L_{1}\left(G_{m} \times G_{m}\right)$. Then

$$
\lim _{n \rightarrow \infty} \sigma_{n}(x, y ; f)=f(x, y) \text { a. e. }(x, y) \in G_{m} \times G_{m} .
$$

\section{Auxiliary Propositions}

Theorem W. (Weisz [22]) Suppose that the operator $T$ is $\sigma$-sublinear and p-quasi-local for each $0<p_{0}<p \leq 1$. If $T$ is bounded from $L_{\infty}\left(G_{m} \times G_{m}\right)$ to $L_{\infty}\left(G_{m} \times G_{m}\right)$, then

$$
\|T f\|_{p} \leq c_{p}\|f\|_{p} \quad\left(f \in H_{p}\left(G_{m} \times G_{m}\right)\right)
$$

for every $0<p_{0}<p<\infty$. In particular for $f \in L_{1}\left(G_{m} \times G_{m}\right)$, holds

$$
\|T f\|_{\text {weak_L } L_{1}\left(G_{m} \times G_{m}\right)} \leq c\|f\|_{1} .
$$

Lemma 1. We have

$$
\begin{gathered}
M_{A} K_{M_{A}}(x, y)=\sum_{k=0}^{A-1} r_{k+1, A-1}(x, y) M_{k} \sum_{r=1}^{m_{k}-1}\left(\sum_{q=0}^{r-1} \psi_{M_{k}}^{q}(x)\right)\left(\sum_{s=0}^{r-1} \psi_{M_{k}}^{s}(y)\right) \\
\times D_{M_{k}}(x) D_{M_{k}}(y)
\end{gathered}
$$




$$
\begin{aligned}
&+\sum_{k=0}^{A-1} r_{k+1, A-1}(x, y) \sum_{r=1}^{m_{k}-1}\left(\sum_{q=0}^{r-1} \psi_{M_{k}}^{q}(x)\right) \psi_{M_{k}}^{r}(y) D_{M_{k}}(x) M_{k} K_{M_{k}}(y) \\
&+\sum_{k=0}^{A-1} r_{k+1, A-1}(x, y) \sum_{r=1}^{m_{k}-1}\left(\sum_{s=0}^{r-1} \psi_{M_{k}}^{s}(y)\right) \psi_{M_{k}}^{r}(x) D_{M_{k}}(y) M_{k} K_{M_{k}}(x) \\
&+r_{1, A-1}(x+y) .
\end{aligned}
$$

Proof of Lemma 1. Since (see [7])

$$
\begin{gathered}
D_{j+r M_{A}}(x)=\left(\sum_{q=0}^{r-1} \psi_{M_{A}}^{q}(x)\right) D_{M_{A}}(x)+\psi_{M_{A}}^{r}(x) D_{j}(x) \\
j=0,1, \ldots, M_{A}-1, r=1,2, \ldots, m_{A-1}-1
\end{gathered}
$$

we can write

$$
\begin{aligned}
& M_{A} K_{M_{A}}(x, y)=\sum_{j=0}^{M_{A}-1} D_{j}(x) D_{j}(y) \\
& =M_{A-1} K_{M_{A-1}}(x, y)+\sum_{r=1}^{m_{A-1}-1} \sum_{j=0}^{M_{A-1}-1} D_{j+r M_{A-1}}(x) D_{j+r M_{A-1}}(y) \\
& =M_{A-1} K_{M_{A-1}}(x, y)+\sum_{r=1}^{m_{A-1}-1} \sum_{j=0}^{M_{A-1}-1}\left(\sum_{q=0}^{r-1} \psi_{M_{A-1}}^{q}(x)\right) D_{M_{A-1}}(x) \\
& \times\left(\sum_{q=0}^{r-1} \psi_{M_{A-1}}^{q}(y)\right) D_{M_{A-1}}(y) \\
& +\sum_{r=1}^{m_{A-1}-1} \sum_{j=0}^{M_{A-1}-1} \psi_{M_{A-1}}^{r}(x) \psi_{M_{A-1}}^{r}(y) D_{j}(x) D_{j}(y) \\
& +\sum_{r=1}^{m_{A-1}-1} \sum_{j=0}^{M_{A-1}-1}\left(\sum_{q=0}^{r-1} \psi_{M_{A-1}}^{q}(x)\right) D_{M_{A-1}}(x) \\
& \times \psi_{M_{A-1}}^{r}(y) D_{j}(y) \\
& +\sum_{r=1}^{m_{A-1}-1} \sum_{j=0}^{M_{A-1}-1}\left(\sum_{s=0}^{r-1} \psi_{M_{A-1}}^{s}(y)\right) D_{M_{A-1}}(y) \\
& \times \psi_{M_{A-1}}^{r}(x) D_{j}(x) \\
& =\left(\sum_{r=0}^{m_{A-1}-1} \psi_{M_{A-1}}^{r}(x+y)\right) M_{A-1} K_{M_{A-1}}(x, y)
\end{aligned}
$$




$$
\begin{aligned}
& +M_{A-1} \sum_{r=1}^{m_{A-1}-1}\left(\sum_{q=0}^{r-1} \psi_{M_{A-1}}^{q}(x)\right)\left(\sum_{s=0}^{r-1} \psi_{M_{A-1}}^{s}(y)\right) D_{M_{A-1}}(x) D_{M_{A-1}}(y) \\
& +\sum_{r=1}^{m_{A-1}-1}\left(\sum_{q=0}^{r-1} \psi_{M_{A-1}}^{q}(x)\right) \psi_{M_{A-1}}^{r}(y) D_{M_{A-1}}(x) M_{A-1} K_{M_{A-1}}(y) \\
& +\sum_{r=1}^{m_{A-1}-1}\left(\sum_{s=0}^{r-1} \psi_{M_{A-1}}^{s}(y)\right) \psi_{M_{A-1}}^{r}(x) D_{M_{A-1}}(y) M_{A-1} K_{M_{A-1}}(x)
\end{aligned}
$$

Iterating this equality we obtain the proof of Lemma 1 .

By results in [17] we have

$$
\left|K_{M_{A}}(x)\right| \lesssim \sum_{s=0}^{A} \frac{M_{s}}{M_{A}} \sum_{x_{s}=1}^{m_{s}-1} D_{M_{A}}\left(x-x_{s} e_{s}\right)
$$

and

$$
n\left|K_{n}(x)\right| \lesssim \sum_{j=0}^{A} M_{j}\left|K_{M_{j}}(x)\right|, M_{A} \leq n<M_{A+1} .
$$

Then from (4) and (5) we can write

$$
\begin{aligned}
n\left|K_{n}(x)\right| & \lesssim \sum_{j=0}^{A} \sum_{s=0}^{j} M_{s} \sum_{x_{s}=1}^{m_{s}-1} D_{M_{j}}\left(x-x_{s} e_{s}\right) \\
& \lesssim \sum_{s=0}^{A} M_{s} \sum_{j=s}^{A} \sum_{x_{s}=1}^{m_{s}-1} D_{M_{j}}\left(x-x_{s} e_{s}\right) .
\end{aligned}
$$

Lemma 2. Let $M_{A} \leq n<M_{A+1}$. Then wee have

$$
\begin{gathered}
n\left|K_{n}(x, y)\right| \lesssim \sum_{j=0}^{A} \sum_{q=0}^{j-1} \sum_{k=q}^{j-1} r_{k+1, j-1}(x, y) \\
\times M_{q} D_{M_{k}}(x) \sum_{y_{q}=1}^{m_{q}-1} D_{M_{k}}\left(y-y_{q} e_{q}\right) \\
+\sum_{j=0}^{A} \sum_{q=0}^{j-1} \sum_{k=q}^{j-1} r_{k+1, j-1}(x, y) \\
\times M_{q} D_{M_{k}}(y) \sum_{x_{q}=1}^{m_{q}-1} D_{M_{k}}\left(x-x_{q} e_{q}\right) \\
+\sum_{j=0}^{A} D_{M_{j}}(x) \sum_{s=0}^{j} M_{s} \sum_{i=s}^{j} \sum_{y_{s}=1}^{m_{s}-1} D_{M_{i}}\left(y-y_{s} e_{s}\right)
\end{gathered}
$$




$$
+\sum_{j=0}^{A} D_{M_{j}}(y) \sum_{s=0}^{j} M_{s} \sum_{i=s}^{j} \sum_{x_{s}=1}^{m_{s}-1} D_{M_{i}}\left(x-x_{s} e_{s}\right) .
$$

proof of Lemma 2. It is proved in [9] that

$$
\begin{aligned}
& n\left|K_{n}(x, y)\right| \lesssim \sum_{j=0}^{A} M_{j}\left|K_{M_{j}}(x, y)\right| \\
& +\sum_{j=0}^{A} D_{M_{j}}(x) \max _{1 \leq n \leq n^{(j)}} n\left|K_{n}(y)\right| \\
& +\sum_{j=0}^{A} D_{M_{j}}(y) \max _{1 \leq n \leq n^{(j)}} n\left|K_{n}(x)\right|,
\end{aligned}
$$

where $n^{(j)}:=\sum_{k=0}^{j} n_{k} M_{k}$.

Then from Lemma 1 and estimation (5) we can write

$$
\begin{aligned}
& n\left|K_{n}(x, y)\right| \lesssim \sum_{j=0}^{A} \sum_{k=0}^{j-1} r_{k+, j-1}(x, y) D_{M_{k}}(x) M_{k}\left|K_{M_{k}}(y)\right| \\
& +\sum_{j=0}^{A} \sum_{k=0}^{j-1} r_{k+1, j-1}(x, y) D_{M_{k}}(y) M_{k}\left|K_{M_{k}}(x)\right| \\
& +\sum_{j=0}^{A} D_{M_{j}}(x) \max _{1 \leq n \leq n^{(j)}} n\left|K_{n}(y)\right| \\
& +\sum_{j=0}^{A} D_{M_{j}}(y) \max _{1 \leq n \leq n^{(j)}} n\left|K_{n}(x)\right| \\
& \lesssim \sum_{j=0}^{A} \sum_{k=0}^{j-1} r_{k+1, j-1}(x, y) D_{M_{k}}(x) \\
& \times \sum_{q=0}^{k} M_{q} \sum_{y_{q}=1}^{m_{q}-1} D_{M_{k}}\left(y-y_{q} e_{q}\right) \\
& +\sum_{j=0}^{A} \sum_{k=0}^{j-1} r_{k+1, j-1}(x, y) D_{M_{k}}(y) \\
& \times \sum_{q=0}^{k} M_{q} \sum_{x_{q}=1}^{m_{q}-1} D_{M_{k}}\left(x-x_{q} e_{q}\right) \\
& +\sum_{j=0}^{A} D_{M_{j}}(x) \sum_{s=0}^{j} M_{s} \sum_{i=s}^{j} \sum_{y_{s}=1}^{m_{s}-1} D_{M_{i}}\left(y-y_{s} e_{s}\right)
\end{aligned}
$$




$$
+\sum_{j=0}^{A} D_{M_{j}}(y) \sum_{s=0}^{j} M_{s} \sum_{i=s}^{j} \sum_{x_{s}=1}^{m_{s}-1} D_{M_{i}}\left(x-x_{s} e_{s}\right) .
$$

Lemma 2 is proved.

\section{Proofs of Main Results}

Proof of Theorem 1. We can write

$$
\begin{aligned}
& \left|\sigma_{n}(x, y ; f)-f(x, y)\right| \\
& \leq \int_{G_{m} \times G_{m}}|f(x-t, y-u)-f(x, y)|\left|K_{n}(t, u)\right| d \mu(t, u) \\
& \leq \frac{c}{n} \sum_{j=0}^{A} \sum_{q=0}^{j-1} \sum_{k=q}^{j-1} \sum_{u_{q}=1}^{m_{q}-1} \int_{G_{m} \times G_{m}}|f(x-t, y-u)-f(x, y)| \\
& \times r_{k+1, j-1}(t, u) M_{q} D_{M_{k}}(t) D_{M_{k}}\left(u-u_{q} e_{q}\right) d \mu(t, u) \\
& +\frac{c}{n} \sum_{j=0}^{A} \sum_{q=0}^{j-1} \sum_{k=q}^{j-1} \sum_{t_{q}=1}^{m_{q}-1} \int_{G_{m} \times G_{m}}|f(x-t, y-u)-f(x, y)| \\
& \times r_{k+1, j-1}(t, u) M_{q} D_{M_{k}}\left(t-t_{q} e_{q}\right) D_{M_{k}}(u) d \mu(t, u) \\
& +\frac{1}{n} \sum_{j=0}^{A} \sum_{s=0}^{j-1} \sum_{i=s}^{j} \sum_{u_{s}=1}^{m_{s}-1} M_{s} \int_{G_{m} \times G_{m}}|f(x-t, y-u)-f(x, y)| \\
& \times \quad D_{M_{j}}(t) D_{M_{i}}\left(u-u_{s} e_{s}\right) d \mu(t, u) \\
& +\frac{c}{n} \sum_{j=0}^{A} \sum_{s=0}^{j-1} \sum_{i=s}^{j} \sum_{t_{s}=1}^{m_{s}-1} M_{s} \int_{G_{m} \times G_{m}}|f(x-t, y-u)-f(x, y)| \\
& \times \quad D_{M_{j}}\left(t-t_{s} e_{s}\right) D_{M_{i}}(u) d \mu(t, u) \\
& =\frac{c}{n} \sum_{j=0}^{A} \sum_{q=0}^{j-1} \sum_{k=q}^{j-1} \sum_{u_{q}=1}^{m_{q}-1} M_{q} M_{k}^{2} \int_{I_{k} \times I_{k}\left(u_{q} e_{q}\right)}|f(x-t, y-u)-f(x, y)| \\
& \times r_{k+1, j-1}(t, u) d \mu(t, u) \\
& +\frac{c}{n} \sum_{j=0}^{A} \sum_{q=0}^{j-1} \sum_{k=q}^{j-1} \sum_{u_{q}=1}^{m_{q}-1} M_{q} M_{k}^{2} \int_{I_{k}\left(t_{q} e_{q}\right) \times I_{k}}|f(x-t, y-u)-f(x, y)| \\
& \times r_{k+1, j-1}(t, u) d \mu(t, u)
\end{aligned}
$$




$$
\begin{gathered}
+\frac{c}{n} \sum_{j=0}^{A} \sum_{s=0}^{j} \sum_{i=s}^{j} \sum_{u_{s}=1}^{m_{s}-1} M_{q} M_{i} M_{j} \\
\times \int_{I_{j} \times I_{i}\left(u_{s} e_{s}\right)}|f(x-t, y-u)-f(x, y)| d \mu(t, u) \\
+\frac{c}{n} \sum_{j=0}^{A} \sum_{s=0}^{j} \sum_{i=s}^{j} \sum_{t_{s}=1}^{m_{s}-1} M_{q} M_{i} M_{j} \\
\times \int_{I_{i}\left(t_{s} e_{s}\right) \times I_{j}}|f(x-t, y-u)-f(x, y)| d \mu(t, u) \\
\leq \frac{c}{n} \sum_{j=0}^{A} M_{j} W_{j}(x, y ; f)
\end{gathered}
$$

which tends to 0 as $n \rightarrow \infty$. This completes the proof of Theorem 1 .

Proof of Theorem 2. Since

$$
V f \leq \sum_{j=1}^{4} V^{(j)} f
$$

by Theorem $\mathrm{W}$, the proof of Theorem 2 will be complete if we show that the operators $V^{(i)}, i=1,2,3,4$ are p-quasi-local for each $1 / 2<p \leq 1$ and bounded from $L_{\infty}\left(G_{m} \times G_{m}\right)$ to $L_{\infty}\left(G_{m} \times G_{m}\right)$.

It follows from (3) that

$$
\|V f\|_{\infty} \leq c\|f\|_{\infty} \sup _{n} \sum_{q=0}^{n} \sum_{k=q}^{n} \frac{M_{q} M_{k}}{M_{k} M_{n}} \leq c\|f\|_{\infty} .
$$

Let $a$ be an arbitrary atom with support $I_{N}\left(z^{\prime}, z^{\prime \prime}\right)=I_{N}\left(z^{\prime}\right) \times I_{N}\left(z^{\prime \prime}\right)$. It is easy to see that $V_{n}(a)=0$ if $n<N$. Therefore we can suppose that $n \geq N$. We may assume that $z^{\prime}=z^{\prime \prime}=0$. Hence

$$
\operatorname{supp}(a) \subset I_{N} \times I_{N} .
$$

Step 1: Integrating over $\bar{I}_{N} \times \bar{I}_{N}$. If $q \geq N$ then $y-u \notin I_{N}$ by (3). Hence

$$
a(x-t, y-u)=0 .
$$

Consequently, we can write

$$
\begin{gathered}
V_{n}^{(1)}(x, y ; a):=\sum_{q=0}^{N-1} \sum_{k=q}^{N-1} \frac{M_{k} M_{q}}{m_{k}} \sum_{t_{q}=1}^{m_{q}-1} \\
\int_{I_{k}\left(t_{q} e_{q}\right) \times I_{k}} a(x-t, y-u) \mathbb{I}_{\left\{t_{r}+u_{r}\left(\bmod m_{r}\right)=0, r=k+1, \ldots, n-1\right\}}(t, u) d \mu(t, u) .
\end{gathered}
$$


Then from (7) $a(x-t, y-u) \neq 0$ implies that

$$
\begin{gathered}
t=\left(0, \ldots, 0, t_{q}, 0, \ldots, 0, x_{k}, \ldots, x_{N-1}, t_{N}, \ldots\right) \\
x=\left(0, \ldots, 0, t_{q}, 0, \ldots, 0, x_{k}, \ldots, x_{N-1}, \ldots\right) \\
u=\left(0, \ldots, 0, y_{k}, m_{k+1}-x_{k+1}, \ldots, m_{N-1}-x_{N-1}, u_{N}, \ldots\right) \\
y=\left(0, \ldots, 0, y_{k}, m_{k+1}-x_{k+1}, \ldots, m_{N-1}-x_{N-1}, y_{N}, \ldots\right) .
\end{gathered}
$$

Hence

$$
\begin{aligned}
\left|V_{n}^{(1)}(x, y ; a)\right| \lesssim & \frac{M_{N}^{2 / p}}{M_{N}^{2}} \sum_{q=0}^{N-1} \sum_{k=q}^{N-1} M_{k} M_{q} \mathbb{I}_{I_{k}\left(t_{q} e_{q}\right)}(x) \\
& \times \mathbb{I}_{I_{N}\left(0, \ldots, 0, y_{k}, m_{k+1}-x_{k+1}, \ldots, m_{N-1}-x_{N-1}\right)}(y)
\end{aligned}
$$

and

$$
\begin{aligned}
\int_{\bar{I}_{N} \times \bar{I}_{N}}\left(V^{(1)}(x, y ; a)\right)^{p} d \mu(x, y) & \leq \frac{c_{p} M_{N}^{2}}{M_{N}^{2 p}} \sum_{q=0}^{N-1} \sum_{k=q}^{N-1} M_{k}^{p} M_{q}^{p} \frac{1}{M_{k}} \frac{1}{M_{N}} \\
& \leq c_{p} \frac{M_{N}}{M_{N}^{2 p}} \sum_{q=0}^{N-1} M_{q}^{p} \sum_{k=q}^{N-1} M_{k}^{p-1} \\
& \leq c_{p}<\infty \quad(1 / 2<p \leq 1) .
\end{aligned}
$$

Analogously, we can prove that

$$
\int_{\bar{I}_{N} \times \bar{I}_{N}}\left(V^{(2)}(x, y ; a)\right)^{p} d \mu(x, y) \leq c_{p}<\infty \quad(1 / 2<p \leq 1) .
$$

Since $x+t \notin I_{N}$ we obtain,

$$
V_{n}^{(3)}(x, y ; a)=0
$$

Analogously, we can prove that

$$
V_{n}^{(4)}(x, y ; a)=0
$$

Combining (3) and (8),11) we obtain

$$
\int_{\bar{I}_{N} \times \bar{I}_{N}}(V(x, y ; a))^{p} d \mu(x, y) \leq c_{p} \quad(1 / 2<p \leq 1) .
$$


Step 2: Integrating over $\bar{I}_{N} \times I_{N}$. Since $V_{n}^{(1)}(x, y ; a)=0$ for $q \geq N$ we have

$$
\text { (13) } \begin{aligned}
& V_{n}^{(1)}(x, y ; a) \\
= & \sum_{q=0}^{N-1} \sum_{k=q}^{N-1} \frac{M_{k} M_{q}}{m_{k}} \sum_{t_{q}=1}^{m_{q}-1} \\
& \quad \int_{I_{k}\left(t_{q} e_{q}\right) \times I_{k}}^{N-1} a(x-t, y-u) \mathbb{I}_{\left\{t_{r}+u_{r}\left(\bmod m_{r}\right)=0, r=k+1, \ldots, n-1\right\}}(t, u) d \mu(t, u) \\
& +\sum_{q=0}^{N-1} \sum_{k=N}^{n} \frac{M_{k} M_{q}}{m_{k}} \sum_{t_{q}=1}^{m_{q}-1} \\
& \int_{I_{k}\left(t_{q} e_{q}\right) \times I_{k}}^{(1,1)} a(x-t, y-u) \mathbb{I}_{\left\{t_{r}+u_{r}\left(\bmod m_{r}\right)=0, r=k+1, \ldots, n-1\right\}}(t, u) d \mu(t, u) \\
= & V_{n}^{(x, 1)}(x, y)+V_{n}^{(1,2)}(x, y ; a) .
\end{aligned}
$$

From (77) $V_{n}^{(1,1)}(x, y ; a) \neq 0$ implies that

$$
\begin{gathered}
u=\left(0, \ldots, 0, u_{N}, \ldots\right) \\
y=\left(0, \ldots, 0, y_{N}, \ldots\right) . \\
t=\left(0, \ldots, 0, t_{q}, 0, \ldots, 0, t_{k}, 0, \ldots, 0, t_{N}, \ldots\right) \\
x=\left(0, \ldots, 0, t_{q}, 0, \ldots, 0, t_{k}, 0, \ldots, 0, x_{N}, \ldots\right)
\end{gathered}
$$

Consequently,

$$
\begin{gathered}
\left|V_{n}^{(1,1)}(x, y ; a)\right| \lesssim \frac{M_{N}^{2 / p}}{M_{N}^{2}} \sum_{q=0}^{N-1} \sum_{k=q}^{N-1} M_{k} M_{q} \sum_{t_{q}=1}^{m_{q}-1} \sum_{t_{k}=1}^{m_{k}-1} \\
\mathbb{I}_{I_{N}\left(e_{q} t_{q}+e_{k} t_{k}\right)}(x) \mathbb{I}_{I_{N}}(y)
\end{gathered}
$$

and

$$
\int_{\bar{I}_{N} \times I_{N}}\left(\sup _{n}\left|V_{n}^{(1,1)}(x, y ; a)\right|\right)^{p} \leq \frac{c_{p} M_{N}^{2}}{M_{N}^{2 p}} \sum_{q=0}^{N-1} \sum_{k=q}^{N-1} M_{k}^{p} M_{q}^{p} \frac{1}{M_{N}^{2}} \leq c_{p} .
$$

From (7) $V_{n}^{(1,2)}(x, y ; a) \neq 0$ implies that

$$
\begin{gathered}
t=\left(0, \ldots, 0, t_{q}, 0, \ldots, 0, t_{k}, \ldots, t_{n-1}, t_{n}, \ldots\right) \\
x=\left(0, \ldots, 0, t_{q}, 0, \ldots, 0, x_{N}, \ldots\right) \\
u=\left(0, \ldots, 0, u_{k}, \alpha_{k+1}, \ldots, \alpha_{n-1}, u_{n}, \ldots\right) \\
y=\left(0, \ldots, 0, y_{N}, \ldots\right)
\end{gathered}
$$

where

$$
\alpha_{j}:=\left\{\begin{array}{c}
m_{j}-t_{j}, t_{j} \neq 0 \\
0, t_{j}=0, j=k+1, \ldots, n-1 .
\end{array}\right.
$$


Thus

$$
\begin{aligned}
\left|V_{n}^{(1,2)}(x, y ; a)\right| & \lesssim \frac{M_{N}^{2 / p}}{M_{N} M_{n}} \sum_{q=0}^{N-1} \sum_{k=q}^{N-1} M_{k} M_{q} \sum_{t_{q}=1}^{m_{q}-1} \mathbb{I}_{I_{N}\left(e_{q} t_{q}\right)}(x) \mathbb{I}_{I_{N}}(y) \\
& \lesssim \frac{c M_{N}^{2 / p}}{M_{n}} \sum_{q=0}^{N-1} M_{q} \mathbb{I}_{I_{N}\left(e_{q} t_{q}\right)}(x) \mathbb{I}_{I_{N}}(y)
\end{aligned}
$$

and $(n \geq N)$

$$
\int_{\bar{I}_{N} \times I_{N}}\left(\sup _{n}\left|V_{n}^{(1,2)}(x, y ; a)\right|\right)^{p} d \mu(x, y) \leq \frac{c_{p} M_{N}^{2}}{M_{N}^{2} M_{N}^{p}} \sum_{q=0}^{N-1} M_{q}^{p} \leq c_{p}<\infty .
$$

Combining (13)-(15) we conclude that

$$
\int_{\bar{I}_{N} \times I_{N}}\left(\left|V^{(1)}(x, y ; a)\right|\right)^{p} d \mu(x, y) \leq c_{p}<\infty .
$$

Let $q<N$. Then it is easy to show that $y-u \notin I_{N}$ and consequently,

$$
\begin{gathered}
a(x-t, y-u)=0, \\
V_{n}^{(2)}(x, y ; a)=0 .
\end{gathered}
$$

Let $q \geq N$. Then $x-t \notin I_{N}$ and

$$
V_{n}^{(2)}(x, y ; a)=0
$$

Hence

$$
V^{(2)}(x, y ; a)=0
$$

Analogously, we can prove that

$$
V^{(4)}(x, y ; a)=0
$$

The estimation of $V_{n}^{(3)}(x, y ; a)$ is analogous to the estimation of $V_{n}^{(1)}(x, y ; a)$ and we can prove that

$$
\int_{\bar{I}_{N} \times I_{N}}\left(\left|V^{(3)}(x, y ; a)\right|\right)^{p} d \mu(x, y) \leq c_{p}<\infty(1 / 2<p \leq 1) .
$$

Combining (16)-(19) we conclude that

$$
\int_{I_{N} \times I_{N}}(|V(x, y ; a)|)^{p} d \mu(x, y) \leq c_{p}<\infty(1 / 2<p \leq 1) .
$$


Step 3: Integrating over $I_{N} \times \bar{I}_{N}$. This case is analogous to the step 2 and we obtain that

$$
\int_{I_{N} \times \bar{I}_{N}}(|V(x, y ; a)|)^{p} d \mu(x, y) \leq c_{p}<\infty \quad(1 / 2<p \leq 1) .
$$

Combining (12), (20) and (21) we complete the proof of Theorem 2 .

\section{REFERENCES}

[1] G. N. Agaev, N.Ya. Vilenkin, G.M. Dzhafarli, and A.I. Rubinshtejn, Multiplicative systems of functions and harmonic analysis on zero-dimensional groups, Baku, Ehlm, 1981 (in Russian).

[2] P. L. Butzer and R. J. Nessel, Fourier Analysis and Approximation, Birkuser, Basel, 1971.

[3] J. Fine, Cesàro summability of Walsh-Fourier series. Proc. Nat. Acad. Sci. USA 41 (1955), 558-591.

[4] G. Gát, Convergence of Marcinkiewicz means of integrable functions with respect to two-dimensional Vilenkin systems. Georgian Math. J. 11 (2004), no. 3, 467-478.

[5] G. Gát and U. Goginava, Almost everywhere strong summability of double WalshFourier series, Journal of Contemporary Mathematical Analysis 50, 1 (2015), 1-13.

[6] G. Gát, U. Goginava and G. Karagulyan, Almost everywhere strong summability of Marcinkiewicz means of double Walsh-Fourier series. Anal. Math. 40 (2014), no. 4, 243-266.

[7] B. I. Golubov, A. V. Efimov, and V. A. Skvortsov, Series and transformations of Walsh, Nauka, Moscow, 1987 (Russian); English transl.: Kluwer Acad. publ; 1991.

[8] U. Goginava, Almost everywhere convergence of $(C, \alpha)$-means of cubical partial sums of $d$-dimensional Walsh-Fourier series. J. Approx. Theory 141 (2006), no. 1, 8-28.

[9] U. Goginava, Marcinkiewicz-Fejér means of double Vilenkin-Fourier series. Studia Sci. Math. Hungar. 44 (2007), no. 1, 97-115.

[10] U. Goginava, The weak type inequality for the Walsh system. Studia Math. 185 (2008), no. 1, 35-48.

[11] U. Goginava and L. Gogoladze, Pointwise summability of Vilenkin-Fourier series. Publ. Math. Debrecen 79 (2011), no. 1-2, 89-108.

[12] U. Goginava and L. Gogoladze, Strong approximation by Marcinkiewicz means of two-dimensional Walsh-Fourier series. Constr. Approx. 35 (2012), no. 1, 1-19.

[13] U. Goginava and F. Weisz, Pointwise convergence of Marcinkiewicz-Fejér means of two-dimensional Walsh-Fourier series. Studia Sci. Math. Hungar. 49 (2012), no. 2, 236-253.

[14] H. Lebesgue. Recherches sur la convergence des séries de Fourier. Math. Annalen, 61:251-280, 1905.

[15] J. Marcinkiewicz, Sur une méthode remarquable de sommation des séries doubles de Fourier. Ann. Scuola Norm. Sup. Pisa, 8:149-160, 1939.

[16] J. Marcinkiewicz and A. Zygmund, On the summability of double Fourier series, Fund. Math. 32 (1939), 122-132.

[17] J. Pál and P. Simon, On a generalization of the concept of derivative. Acta Math. Acad. Sci. Hungar. 29 (1977), no. 1-2, 155-164.

[18] H. G. Feichtinger and F. Weisz, Wiener amalgams and pointwise summability of Fourier transforms and Fourier series, Math. Proc. Comb. Phil. Soc, 140 (2006), 509536.

[19] F. Schipp, Über gewissen maximaloperatoren, Ann. Univ. Sci. Budapest Sect. Math., 18(1975), 189-195. 
[20] F. Weisz, Convergence of singular integrals., Ann. Univ. Sci. Budapest Sect. Math. 32 (1989), 243-256.

[21] F. Weisz, Convergence of double Walsh-Fourier series and Hardy spaces. Approx. Theory 8 its. Appl. 17, 2(2001), 32-44.

[22] F. Weisz, Summability of multi-dimensional Fourier series and Hardy space, Kluwer Academic, Dordrecht, 2002.

[23] F. Weisz. Walsh-Lebesgue points of multi-dimensional functions. Anal. Math., 34:307$324,2008$.

[24] L. Zhizhiashvili, A generalization of a theorem of Marcinkiewicz. Math. USSR, Izvestija, 2:1065-1075, 1968. (in Russian).

[25] L. Zhizhiashvili, Trigonometric Fourier Series and their Conjugates. Kluwer Academic Publishers, Dordrecht, 1996.

[26] A. Zygmund. Trigonometric Series. Cambridge Press, London, 3rd edition, 2002.

U. Goginava, Department of Mathematics, Faculty of Exact and Natural Sciences, Ivane Javakhishvili Tbilisi State University, Chavchavadze str. 1, TBILISI 0128, Georgia

E-mail address: zazagoginava@gmail.com 